\title{
Modeling and Controller Design of a Maglev Guiding System for Application in Precision Positioning
}

\author{
Mei-Yung Chen, Student Member, IEEE, Ming-Jyh Wang, and Li-Chen Fu, Senior Member, IEEE
}

\begin{abstract}
In this paper, the authors analyze the dynamics of a magnetic guiding system and derive its analytical model with full degrees of freedom (DOFs). Then, an adaptive controller which deals with unknown parameters is proposed to regulate the five DOFs in this system. The guiding system, including sensors and driver subsystems, has actually been implemented. Based on the experimental results, satisfactory performance including stiffness and resolution has been achieved. This validates the design of the system hardware and demonstrates the feasibility of the developed controller.
\end{abstract}

Index Terms-Adaptive control, guideway transportation, magnetic levitation (maglev), motion control.

\section{INTRODUCTION}

$\mathbf{R}$ ECENTLY, magnetic levitation has been considered as one of the most suitable ways to achieve high-precision transportation. According to Hollis et al.. [1], [2], it creates a stable state without any mechanical contact when the gravitational force is solely counterbalanced by magnetic forces. Of course, such contact-free levitation has to be enforced for all degrees of freedom (DOFs) of the rigid body.

Often, a distinction is made between magnetic suspension and magnetic levitation. The former refers to systems based on attractive magnetic forces whereas the latter refers to those based on repulsive forces. However, this restricted sense of the two terms does not cover all types of contact-free magnetic support. Therefore, it is the current trend to use the term "levitation" in a more general sense and to abbreviate "magnetic levitation" as "maglev."

Previous work on maglev systems has spanned many fields. A large volume of literature has been published. Some wellknown fields include maglev transportation [3], [4], wind tunnel levitation [5], magnetic bearings [6], antivibration tables [7], and photolithography stepper [8]. Here, however, this paper will only investigate maglev techniques used in the field of shortrange travel with precision positioning. In this paper, a prototype maglev system has been designed and implemented to verify its high level of performance.

Manuscript received November 19, 2001; revised September 20, 2002. Abstract published on the Internet March 4, 2003. This work was supported by the National Science Council, R.O.C., under Grant NSC-90-2213-E-002-146.

M.-Y. Chen is with R242, Department of Electrical Engineering, National Taiwan University, Taipei, Taiwan, R.O.C. (e-mail:cmy@cc.ee.ntu.edu.tw).

M.-J. Wang is with Chung-Shan Institute of Science and Technology, Taoyuan, Taiwan, R.O.C.

L.-C. Fu is with R242, Department of Electrical Engineering, National Taiwan University, Taipei, Taiwan, R.O.C., and also with R533, Department of Computer Science and Information Engineering, National Taiwan University, Taipei, Taiwan, R.O.C. (e-mail:lichen@ccms.ntu.edu.tw).

Digital Object Identifier 10.1109/TIE.2003.812354
In general, maglev systems can be classified into two categories according to whether the utilized magnetic forces are attractive or repulsive, each type having various kinds of possible arrangements. Notably, the gravity of the carrier in a permanent maglev system is counterbalanced by the magnetic force generated by the opposing magnetic fields between the permanent magnets on the carrier and the electromagnets inside the guiding tracks, so that very slight power consumption is required for the carrier levitation. However, unlike the attractive case, a repulsive maglev is vertically stable but laterally unstable. Therefore, an appropriate mechanical design with additional electromagnets is necessary to provide proper guiding control of the carrier. The maglev silicon wafer transporter designed by Wang [9] is an example of a design which applies the aforementioned technique.

On account of these factors, permanent magnets are used in the maglev system for levitation, and electromagnets are used for positioning and stabilization. In this paper, a prototype maglev guiding system is introduced, whose underlying concept is similar to those in [9] and [10]. However, the latter works only developed 2-DOF (translation and rotation) controllers, and the control laws were based on traditional control methods. These developed controllers may not meet the precision control purpose for the maglev systems, because these systems naturally are subject to many uncertainties. For example, coil impedance varies with temperature fluctuation, different payload is put on the carrier, and so on. On the other hand, this system has a natural magnetic damping in the other three dimensions (vertical, roll, and pitch). Despite the existence of the magnetic damping, lack of control of those dimensions will, however, lead to a long settling time of the entire system. Hence, there are several features of the system developed in this paper, namely, decrease of energy dissipation, increase of load capacity, derivation of a complete 5-DOF analytical model (i.e., without considering the degree of long-range actuation), and highprecision adaptive controller. In particular, it is worth mentioning that the hereby developed controller adopts a nontraditional adaptive control approach. Experimental results are presented here which demonstrate the feasibility of the proposed guiding system.

The organization of this paper is as follows. In Section II, some magnetic formulas will be reviewed, and then the design aspects of the implemented prototype system are described and the mathematical model is presented in detail. In Section III, a sound adaptive controller for the prototype maglev system is given. Section IV presents extensive simulation and experimental results which demonstrate the effectiveness of the system design and its controller. Some discussion is also included in Section IV. Finally, conclusions are drawn in Section V. 


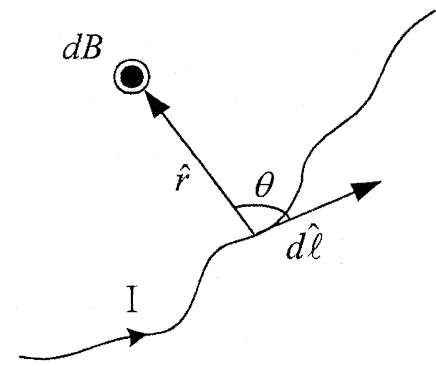

Fig. 1. Magnetic flux density from arbitrary current-carrying wire.

\section{SYSTEM DESCRIPTION AND MODELING}

In this section, some magnetic formulas will be reviewed, which will be used in the model derivation. Then, the mechanical design of a maglev guiding system will be described. The full-DOFs analytical model of the design system will be thoroughly derived and investigated.

\section{A. Preliminary}

According to Biot-Savart's Law [13], the magnetic flux density generated from an arbitrary current-carrying wire is

$$
d \mathbf{B}=\frac{\mu_{0} I}{4 \pi} \frac{d \ell \times \hat{r}}{r^{2}}
$$

where $\mu_{0}$ denoting the permeability in a free space is equal to $4 \pi \times 10^{-7} \mathrm{H} / \mathrm{m}, r$ is the distance from wire to dipole moment, and $I$ is the magnitude of the current flowing through the wire. Then, from Fig. 1, (2.1) can rewritten as follows:

$$
d \mathbf{B}=\frac{\mu_{0} I}{4 \pi} \frac{d \ell \sin \theta}{r^{2}} .
$$

Thus, the magnetic flux density from a straight infinity-length current-carrying wire is

$$
\mathbf{B}=\frac{\mu_{0} I}{4 \pi} \int_{\ell=-\infty}^{\ell=\infty} \frac{d \ell \sin \theta}{r^{2}} .
$$

After arranging (2.3), the magnetic flux density can be obtained as

$$
\mathbf{B}=\frac{\mu_{0} I}{2 \pi r} .
$$

Then, according to Ampere's circuit law, the magnetic flux in any point around an infinitely long current-carrying straight wire at a point $(x, z)$ as shown in Fig. 2 can be obtained as

$$
\mathbf{B}=\frac{\mu_{0} I}{2 \pi}\left(\frac{-z}{x^{2}+z^{2}} \hat{i}+\frac{x}{x^{2}+z^{2}} \hat{k}\right)
$$

where $\hat{i}$ and $\hat{k}$ are the unit vectors in the Cartesian coordinate.

Without the presence of an electrical field, the expression of the Lorentz force [13] for a permanent magnet of $\mathbf{u}$ can be simplified as

$$
\mathbf{F}=(\mathbf{u} \cdot \nabla) \mathbf{B}
$$

where $\mathbf{u}$ is the dipole moment of this infinitesimal current loop.

Since the magnetic field originating from an infinitesimal permanent magnet is equivalent to the field from an infinitesimal current loop, the force applied to an infinitesimal magnet can be obtained directly from (2.6). For an understanding of the Lorentz force applied to a magnet, (2.6) can be expanded into two equations corresponding to forces in different Cartesian coordinate axes, namely,

$$
\begin{aligned}
& F_{x}=\mathbf{u} \cdot \nabla B_{x} \\
& F_{z}=\mathbf{u} \cdot \nabla B_{z} .
\end{aligned}
$$

These equations indicate that the forces applied to a magnet are proportional to the magnetic field gradients. In other words, no force exists in a uniform magnetic field. Nevertheless, the torques applied to a magnet are not subject to this constraint. They exist as long as the filed is present, and the torques applied to a magnet are given by

$$
\mathbf{T}=\mathbf{u} \times \mathbf{B} .
$$

Combining (2.5) and (2.7)-(2.9), assuming the dipole lies in the $Z$ direction, the equations of the forces and torques on a magnet induced by an infinitely long current-carrying straight wire can be rewritten as

$$
\begin{aligned}
F_{x}(x, z, I) & =\frac{\mu_{0} I u_{z}}{2 \pi} \frac{\left(z^{2}-x^{2}\right)}{\left(x^{2}+z^{2}\right)^{2}} \hat{i} \\
F_{z}(x, z, I) & =\frac{\mu_{0} I u_{z}}{\pi} \frac{-x z}{\left(x^{2}+z^{2}\right)^{2}} \hat{k} \\
T(x, z, I) & =\frac{\mu_{0} I u_{z}}{2 \pi} \frac{z}{x^{2}+z^{2}} \hat{k} \equiv T(x, z, I) \hat{k} .
\end{aligned}
$$

\section{B. Maglev Guiding System}

For a stepper in the semiconduction environment, one always finds a linear sliding mechanism to support and to guide the carrier. The system proposed here is by an active control which provides a contactless sliding mechanism as previously mentioned, which, however, is not equipped with the kind of magnetic bearing of a rotating machine. For the detailed design of the system, the reader can refer to [11]. In this section, only a brief description will be given. The features of this maglev guiding system include: 1) repulsive levitation; 2) hybrid magnets; 3 ) a passive carrier and active track; 4) an oblong coil concept; and 5) a four-track design.

In order to control 3 DOFs in attitude and 2 DOFs in lateral displacement (except the one for long-range propulsion), i.e., a total of 5 DOFs of the carrier separately, a four-track design is sufficient to supply such 5-DOFs control. In addition, there are two other advantages. One is that the overall structure can become symmetric, which facilitates employment of a linear actuator to realize the long-range propulsion. Another is that it can be more robust to the payload arrangement on the carrier, which may not necessarily be so symmetric.

The paper chooses repulsive levitation instead of attractive levitation for several reasons. First, in an attractive system, the carrier floats because its "feet" are suspended beneath the tracks. Hence, some connecting structural components between the carrier body and the carrier feet are needed to support the carrier to move above the tracks. These extra structural components, unless carefully designed, will easily weaken the 


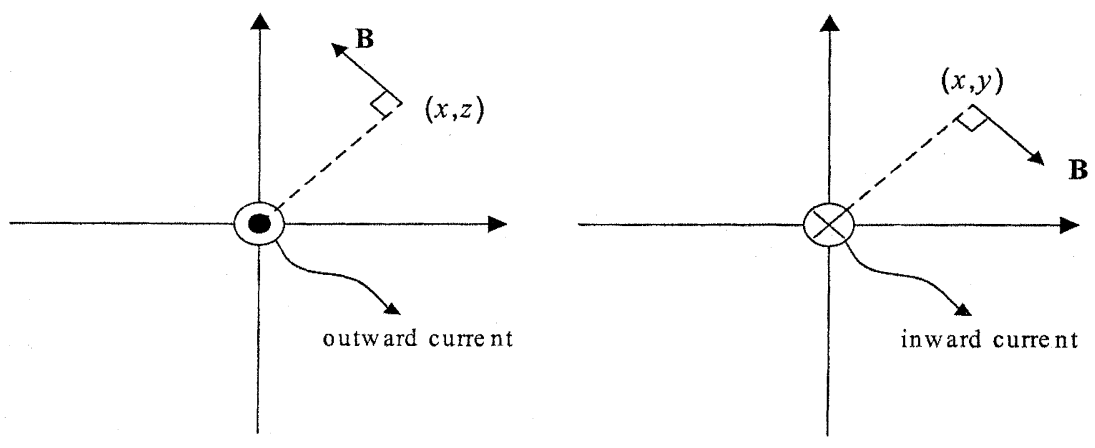

Fig. 2. Directions of $\mathbf{B}$ for opposite directions of current.

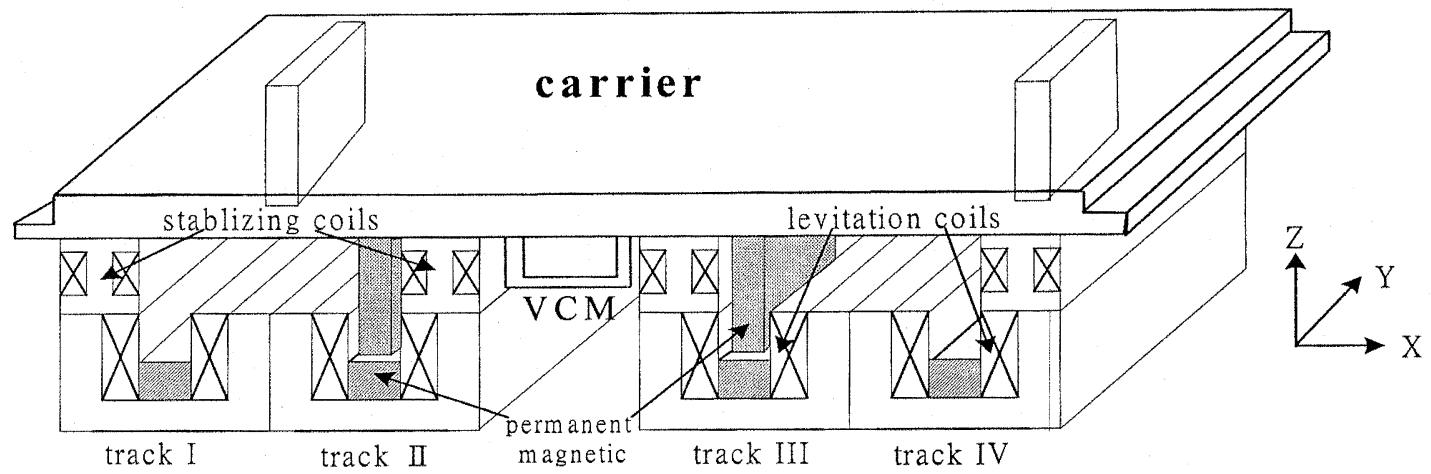

Fig. 3. Maglev guiding system.

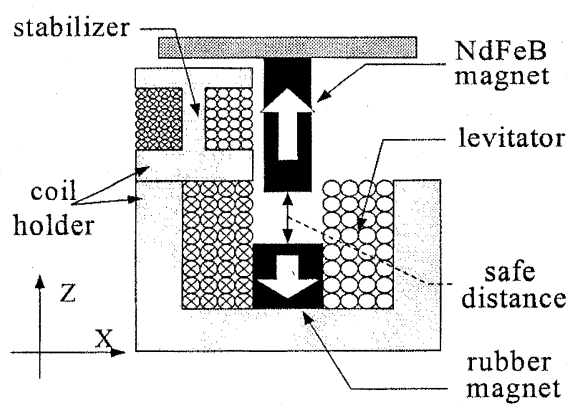

Fig. 4. Straight-cut face of the track.

rigidity of the overall carrier structure. Second, the aforementioned components will increase the carrier weight. However, the carrier can naturally move above the repulsive tracks. The overall system is shown in Fig. 3.

1) Track and Carrier Design: The tracks together must provide a levitation force to counteract the carrier gravity. Also, due to the nature of vertical instability of a repulsive system, stabilizers are needed inside. Fig. 4 shows the straight-cut face of the track. The stabilizer can control the lateral position of a levitating $\mathrm{NdFeB}$ magnet whereas the levitator can control the vertical (up-down) position of a levitating $\mathrm{NdFeB}$ magnet. However, in fact, there are two sources of levitation forces, one from the just mentioned coils and the other from the rubber magnet affixed to the track. The rubber magnet is used to provide the main levitation force whereas the coils fine tune the vertical positioning so as to control the carrier.

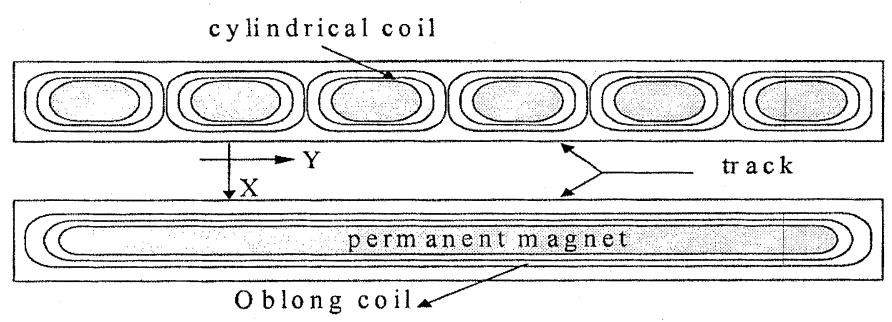

Fig. 5. Cylindrical coils versus an oblong coil.

In order to provide a uniform magnetic field along the large moving range, the tracks using an oblong coil with a permanent magnet inside has been designed as shown in Fig. 5. The tracks lengths are $400 \mathrm{~mm}$. Some maglev trains use a series of cylindrical coils to generate the magnetic field along the long traveling range, but nonuniform magnetic fields may exist at the connections of every pair of neighboring coils.

The carrier is made of acrylic material with dimensions of $270 \mathrm{~mm} \times 200 \mathrm{~mm} \times 8 \mathrm{~mm}$. An area of $3 \mathrm{~mm} \times 35 \mathrm{~mm}$ on each side of the carrier is used as a reference plane for vertical sensors. Four NdFeB magnets, each with dimensions of $7 \mathrm{~mm} \times 50 \mathrm{~mm} \times 25 \mathrm{~mm}$, are attached under the carrier and are placed close to its four corners.

2) Operation Principle: The lateral position of the carrier can actually be controlled by the stabilizers. The four stabilizing coils are grouped into two sets: inner tracks and outer tracks. Then, the principle shown in Figs. 6 and 7 to control rotation of the carrier about a vertical axis and its lateral translation of the carrier can be used. 


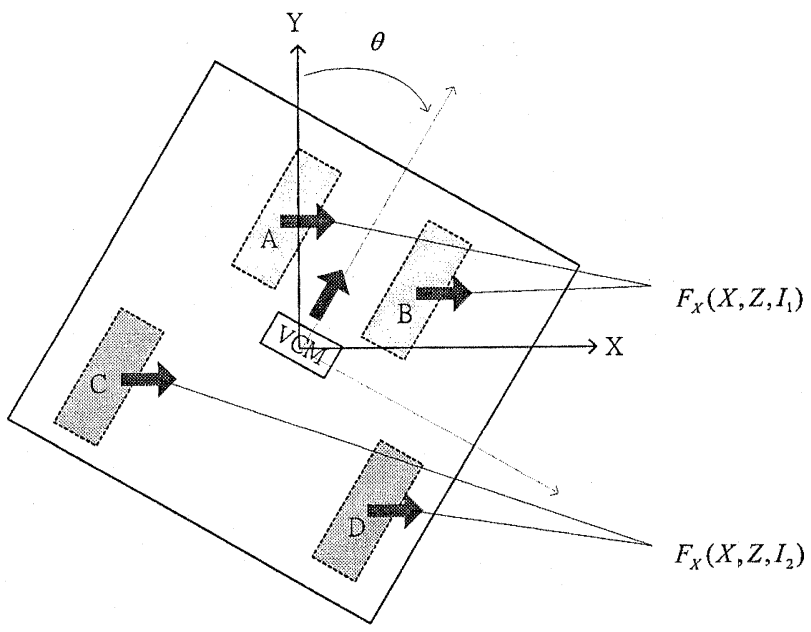

Fig. 6. Stabilizing control forces acting on the magnets.

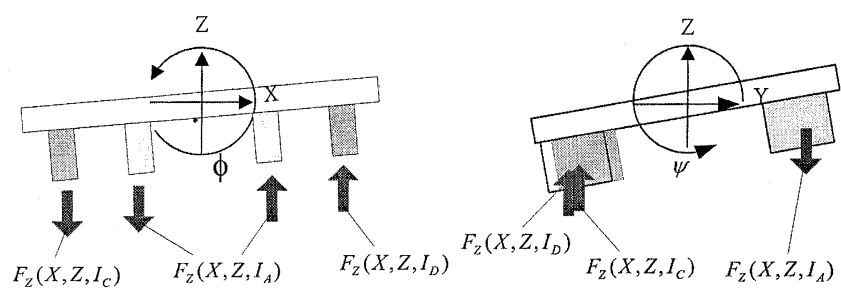

Fig. 7. Levitating control forces acting on the magnets.

Letting the direction pointing upwards be the $z$ direction and the direction along the long-range travel be the $y$ direction, then the lateral direction is naturally the $x$ direction. Thus, the former control $\left(I_{1}, I_{2}\right)$ setup takes care of the translational $(X)$ freedom in the $x$ direction and the rotational $(\theta)$ freedom in the $z$ direction (referred to as yawing). In order to deal with the single DOF $(Z)$ in the $z$ direction and the other two DOFs $(\psi, \phi)$ in attitude, the two levitation coils associated with tracks II and III are further made as a group as shown in Fig. 3 into which a single control $I_{A}$ is applied, and the levitation coils in track I and track IV are independently controlled by separate currents, $I_{C}$ and $I_{D}$, respectively. By this kind of setup, the total four levitation coils can provide another 3 DOFs control, covering the $y$-axis rotation (referred to as rolling), $x$-axis rotation (referred to as pitching), and $z$-direction translation.

Via the stabilizers and levitators, 5 out of the 6 DOFs of the carrier are well controlled, leaving only one direction left to be controlled over a long range. It should be clear that, whether the high-precision positioning performance can be achieved or not depends on how well the guiding system can be controlled. To provide the actuation force in the $y$ direction, a modified voice coil motor (VCM) is employed.

\section{Analytical Model}

In order to achieve the objective of high-precision positioning, an appropriate controller has to be developed. Thus, a complete analytical model, characterizes the dynamics of the aforementioned 5 DOF's, is imperative for designing a good controller.
Before starting the modeling process, several assumptions must be made for the purpose of simplification.

Assumption A Referring to Fig. 4, the $n$-turn wires of stabilizing and levitating coils that are distributed over the cross section of each track are lumped together as an equivalent single wire whose cross section is located at the center of that of the $n$-turn wires.

Assumption B Each levitator permanent magnet (rubber magnet located inside a track) is viewed as a single turn of current-carrying wire wound around the side surface of the magnet.

Assumption C The tracks are long enough with respect to the travel range of the carrier so that all the levitation magnets (right beneath the carrier) are only subject to uniform magnetic fields.

Assumption D Each levitation magnet is considered as one single magnetic dipole carrying the same magnetic dipole moment and is located at the center of each magnet.

Assumption E The magnetic forces and torques between the levitation magnets and levitators can be linearized about the small displacements in the $x$ direction and $z$ direction. This property can also be applied to the interaction situation between the levitation magnets and the stabilizing coils.

In assumption $\mathrm{A}$, the force and torque characteristics of the lumped model and the original model are not identical but are very similar [9]. In assumption B, the volume current density of the permanent magnet is zero if it is uniformly magnetized; hence, only a surface current exists. Following assumption A, assumption B is indirectly implied. Assumption C allows the magnetic field to be simply described by (2.5). Assumption D makes it feasible to employ (2.10)-(2.12) to derive the nonlinear interaction forces and torques in this system. Finally, assumption $\mathrm{E}$ is reasonable when the mechanical structure is well designed. For empirical justification, the magnetic force acting on the permanent magnet fixed beneath the carrier shows in Fig. 9.

1) Dynamics of a Carrier: In carrier design, the material is chosen such that the rigidity of the carrier can be ensured. To understand the dynamics of a rigid body, it is necessary to know how to describe an arbitrary orientation of a rigid body in space. Here, the $z-y-x$ Eulerian angle orientational representation will be adopted. Let the $x y z$ coordinate system be fixed on the carrier, and let the $X Y Z$ coordinate system be fixed on the track, as shown in Fig. 8. If these two coordinate systems coincide initially, an arbitrary orientation of the $x y z$ coordinate system can be described by the following rotational sequence:

1) a positive rotation $\theta$ with respect to the $Z$ axis, resulting in an $x^{\prime} y^{\prime} z^{\prime}$ system;

2) a positive rotation $\phi$ with respect to the $y^{\prime}$ axis, resulting in an $x^{\prime \prime} y^{\prime \prime} z^{\prime \prime}$ system;

3 ) a positive rotation $\psi$ with respect to the $x^{\prime \prime}$ axis, resulting in an $x y z$ system;

where $\theta, \phi$ and $\psi$ are the Eulerian angles of the $x y z$ coordinate system, or of the carrier. 


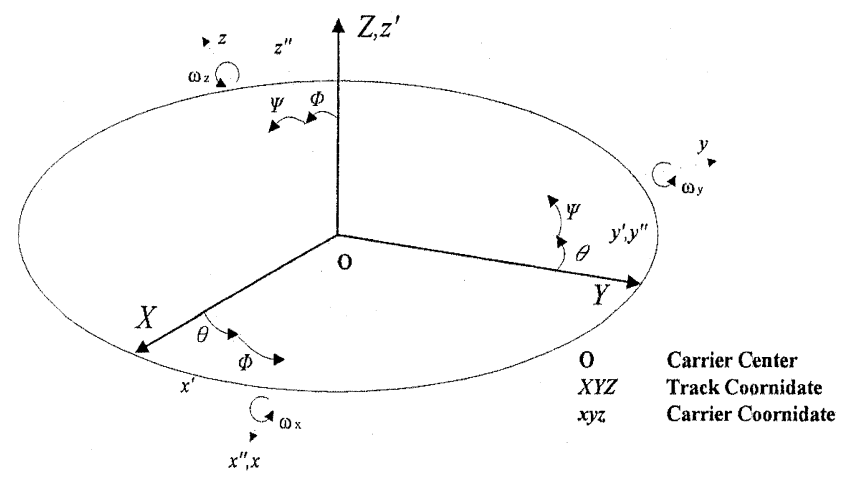

Fig. 8. Eulerian angles.

Consider a carrier represented by a uniform box-shaped object with the center of mass coincident with the center of geometry. Assume rather small displacements in rotation and translation, and neglect the higher order terms. Based on the Newton-Euler method, the equation of motion can be obtained as

$$
F_{X}=m \ddot{X} \quad F_{Z}=m \ddot{Z}
$$

where $F_{X}$ and $F_{Z}$ are the resultant forces acting on the carrier along the $X$ axis and $Z$ axis and $m$ is the mass of the carrier. Moreover,

$$
T_{x}=I_{x} \ddot{\psi}, \quad T_{y}=I_{y} \ddot{\phi}, \quad T_{z}=I_{z} \ddot{\theta}
$$

where $T_{x}, T_{y}$ and $T_{z}$ are the external torques, exerted on the carrier and are represented with respect to the $x y z$ coordinate, and $I_{x}, I_{y}$, and $I_{z}$ are the principal moments of inertia of the carrier.

However, to derive the external torques and forces acting on the carrier relative to the $X Y Z$ coordinate system, transformation from $T_{x}, T_{y}$, and $T_{z}$ to $T_{X}, T_{Y}$, and $T_{Z}$ is necessary. Applying transformation and the small angle assumption, the transformation can be simplified as

$$
\begin{aligned}
& T_{z}=T_{Z}+T_{X} \phi-T_{Y} \psi \\
& T_{y}=T_{Y}-T_{X} \theta+T_{Z} \psi \\
& T_{x}=T_{X}+T_{Y} \theta-T_{Z} \phi .
\end{aligned}
$$

Performing variational calculus on these equations with respect to the origin, one obtains

$$
\begin{aligned}
I_{z} \delta \ddot{\theta} & =\delta T_{Z}+\left.T_{X}\right|_{0} \delta \phi-\left.T_{Y}\right|_{0} \delta \psi \\
I_{y} \delta \ddot{\phi} & =\delta T_{Y}-\left.T_{X}\right|_{0} \delta \theta+\left.T_{Z}\right|_{0} \delta \psi \\
I_{x} \delta \ddot{\psi} & =\delta T_{X}+\left.T_{Y}\right|_{0} \delta \theta-\left.T_{Z}\right|_{0} \delta \phi .
\end{aligned}
$$

Observing the mechanical design, $\left.T_{X}\right|_{0}=\left.T_{Y}\right|_{0}=\left.T_{Z}\right|_{0}=$ 0 can be obtained. Therefore, the rotational equations of motion are linearized to

$$
I_{z} \delta \ddot{\theta}=\delta T_{Z} \quad I_{y} \delta \ddot{\phi}=\delta T_{Y} \quad I_{x} \delta \ddot{\psi}=\delta T_{X} .
$$

Equations (2.13) and (2.17) result in the dynamics of the carrier.

2) Magnetic Force Between the Levitation Magnet and Tracks: The interaction force between the levitation magnet and levitator in the $X$ direction can be obtained from (2.10). There exist only small displacements $\delta X$ and $\delta Z$ as shown in

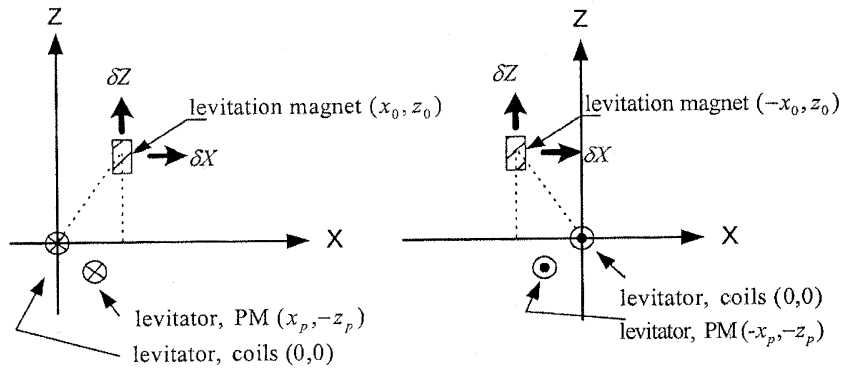

Fig. 9. Force diagram from the levitator.

Fig. 9. Define $F_{X C}$ as the $X$-direction force contributed by physical coils, $F_{X M}$ is the $X$-direction force contributed by the rubber magnet, $I_{p}$ is the equivalent current of the rubber magnet, and the positive direction of current is beyond the scope of this paper. The magnetic force exerted on the levitation magnet can perform linearization about $x$ and $z$ such that

$$
\begin{aligned}
F_{X L}= & F_{X C}+F_{X M} \cong\left[-\frac{4 \mu_{0} I_{0}}{\pi} \frac{x_{0}^{3}-3 x_{0} z_{0}^{2}}{\left(x_{0}^{2}+z_{0}^{2}\right)^{3}}\right. \\
& \left.-\frac{4 \mu_{0} I_{p}}{\pi} \frac{\left(x_{0}-x_{p}\right)^{3}-3\left(x_{0}-x_{p}\right)\left(z_{0}+z_{p}\right)^{2}}{\left(\left(x_{0}-x_{p}\right)^{2}+\left(z_{0}+z_{p}\right)\right)^{3}}\right] u_{z} \delta X \\
& +\left[\frac{2 \mu_{0}}{\pi} \frac{z_{0}^{2}-x_{0}^{2}}{\left(x_{0}^{2}+z_{0}^{2}\right)^{2}}\right] u_{z} \delta I \\
\equiv & K_{F X L} \cdot \delta X+K_{I X L} \cdot \delta I
\end{aligned}
$$

where $x_{0}<z_{0}$ and $I=I_{0}+\delta I$, which implies that $K_{F X L}>0$ and $K_{I X L}>0$. In a similar way, the interaction force between the levitation magnet and the levitator in the $Z$ direction can be obtained as

$$
\begin{aligned}
F_{Z L} \cong & {\left[\frac{4 \mu_{0} I_{0}}{\pi} \frac{x_{0} z_{0}}{\left(x_{0}^{2}+z_{0}^{2}\right)^{2}}\right.} \\
& \left.+\frac{4 \mu_{0} I_{p}}{\pi} \frac{\left(x_{0}-x_{p}\right)\left(z_{0}+z_{p}\right)}{\left(\left(x_{0}-x_{p}\right)^{2}+\left(z_{0}+z_{p}\right)^{2}\right)^{2}}\right] u_{z} \\
& +\left[\frac{4 \mu_{0} I_{0}}{\pi} \frac{x_{0}^{3}-3 x_{0} z_{0}^{2}}{\left(x_{0}^{2}+z_{0}^{2}\right)^{3}}\right. \\
& \left.+\frac{4 \mu_{0} I_{p}}{\pi} \frac{\left(x_{0}-x_{p}\right)^{3}-3\left(x_{0}-x_{p}\right)\left(z_{0}+z_{p}\right)^{2}}{\left(\left(x_{0}-x_{p}\right)^{2}+\left(z_{0}+z_{p}\right)^{2}\right)^{3}}\right] u_{z} \delta Z \\
\equiv & K_{K Z L}+K_{F Z L} \delta Z
\end{aligned}
$$

where $K_{K Z L}>0$ and $K_{F Z L}>0$.

The interaction torque between the levitation magnet and the levitator can be derived from (2.12). After simplification, one can obtain

$$
\begin{aligned}
T_{Y L} \cong & {\left[-\frac{8 \mu_{0} I_{0}}{\pi} \frac{x_{0} z_{0}}{\left(x_{0}^{2}+z_{0}^{2}\right)^{2}}-\frac{8 \mu_{0} I_{p}}{\pi}\right.} \\
& \left.\cdot \frac{\left(x_{0}-x_{p}\right)\left(z_{0}+z_{p}\right)}{\left(\left(x_{0}-x_{p}\right)^{2}+\left(z_{0}+z_{p}\right)^{2}\right)^{2}}\right] u_{z} \delta X \\
& +\left[\frac{z_{0}}{x_{0}^{2}+z_{0}^{2}}\right] u_{z} \delta I \\
\equiv & K_{T Y L} \cdot \delta X+K_{I Y L} \cdot \delta I
\end{aligned}
$$

where $x_{0}<z_{0}$, which implies that $K_{T Y L}>0$ and $K_{I Y L}>0$. 


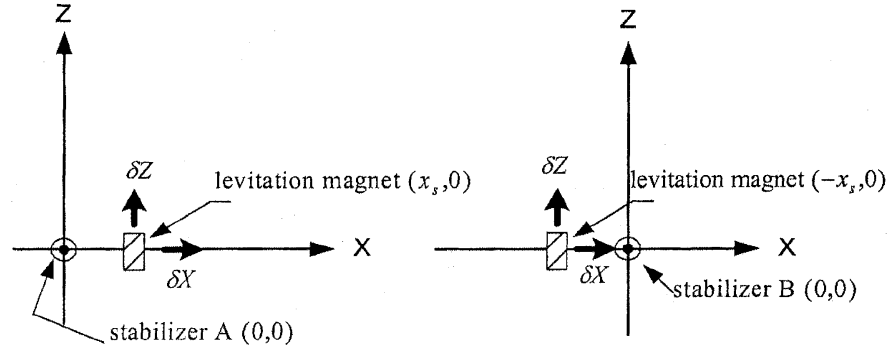

Fig. 10. Force diagram from outer tracks of the stabilizer.

It should be noted that $K_{F X L}>0$ means the lateral direction is unstable in terms of force versus displacement whereas $F_{F Z L}<0$ means the vertical direction is stable also in terms of force versus displacement. These two phenomena indicate the property of the repulsive maglev system: vertically stable but laterally unstable.

The stabilizers can be classified into two groups: one with the inner tracks and the other with the outer tracks. Here, the stabilizers associated with the outer tracks will be considered as shown in Fig. 8, and similar results will also hold for those with inner tracks.

The interaction force between the levitation magnet and the stabilizer A in the $X$ direction can be derived from (2.10). Applying linearization to the function $F_{x}(x, z, I)$ about $x=0$ and $z=0$ subject to small displacements, $\delta X$ and $\delta Z$, as shown in Fig. 10, which can be simplified as

$$
\begin{aligned}
F_{X A S} \cong & \left(\frac{-\mu_{0} I_{0}}{2 \pi} \frac{1}{x_{s}^{2}}\right) u_{z}+\left(\frac{\mu_{0} I_{0}}{\pi} \frac{1}{x_{s}^{3}}\right) u_{z} \delta X \\
& +\left(\frac{-\mu_{0} I_{0}}{2 \pi} \frac{1}{x_{s}^{2}}\right) u_{z} \delta I \\
\equiv & K_{K A S}+K_{F X S} \delta X+K_{I X S} \delta I
\end{aligned}
$$

where $K_{K A S}<0, K_{F X S}>0$, and $K_{I X S}<0$. By the same token, the remaining force and torque on the levitation magnet exerted by the stabilizer can be obtained.

3) Movement of Magnets: The magnetic forces exerted on the levitation magnets of the carrier will be derived in the following. The displacements of the levitation magnets must be sensed first in order to calculate these forces. The relative positions of the levitation magnets are as shown in Fig. 11. The coordinates with respect to the $x y z$ coordinate system are, respectively, as follows:

$$
A\left(-b_{1}, a, 0\right) \quad B\left(b_{1}, a, 0\right) \quad C\left(-b_{2},-a, 0\right) \quad D\left(b_{2},-a, 0\right)
$$

where $A, B, C$, and $D$ denote the center positions of these magnets.

Therefore, the $x y z$-coordinate system into the $X Y Z$-coordinate system will be transformed as

$$
\begin{aligned}
& A_{X Y Z}=T^{T}(\theta, \phi, \psi) A_{x y z} \\
& B_{X Y Z}=T^{T}(\theta, \phi, \psi) B_{x y z} \\
& C_{X Y Z}=T^{T}(\theta, \phi, \psi) C_{x y z} \\
& D_{X Y Z}=T^{T}(\theta, \phi, \psi) D_{x y z}
\end{aligned}
$$

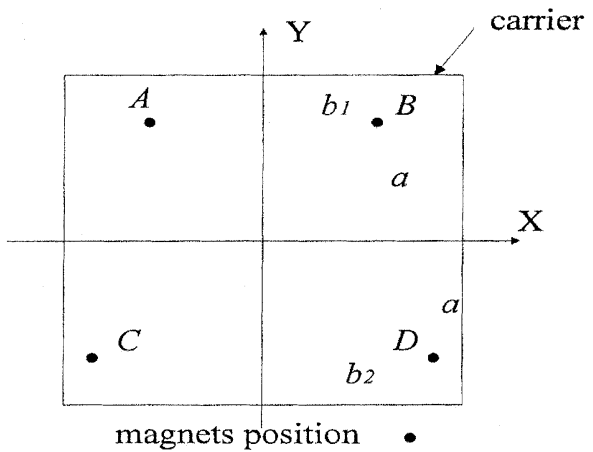

Fig. 11. Positions of levitation magnets.

where $T$ is a transformation matrix with the assumption of small angle displacement. In additional to $X$ and $Z$ translation, the positions of the magnets in the $X Y Z$-coordinate system are

$$
\begin{aligned}
& A_{X Y Z}=\left[\begin{array}{l}
-b_{1}-a \theta+X \\
a-b_{1} \theta \\
b_{1} \phi+a \psi+Z
\end{array}\right] \\
& B_{X Y Z}=\left[\begin{array}{l}
b_{1}-a \theta+X \\
a+b_{1} \theta \\
-b_{1} \phi+a \psi+Z
\end{array}\right] \\
& C_{X Y Z}=\left[\begin{array}{l}
-b_{2}+a \theta+X \\
-a-b_{2} \theta \\
b_{2} \phi-a \psi+Z
\end{array}\right] \\
& D_{X Y Z}=\left[\begin{array}{l}
b_{2}+a \theta+X \\
-a+b_{2} \theta \\
-b_{2} \phi-a \psi+Z
\end{array}\right] .
\end{aligned}
$$

4) Equations of Motion: By substituting the positions of the levitation magnet as in (2.24) into the force equations and torque equations in (2.18)-(2.21), one can get the forces and torques exerted on each levitation magnet. Next, one can substitute these forces and torques equations into the dynamics of the carrier, and then one can obtain the model of this maglev guiding system. Before this, one should define the control current in the inner stabilizer as $I_{1}$, whereas in the outer stabilizer it is $I_{2}$. Control currents in levitators corresponding the levitation magnets $\mathrm{A}, \mathrm{B}, \mathrm{C}$, and D are $I_{A}, I_{B}, I_{C}$, and $I_{D}$, respectively. Let $I_{A 0}$ and $I_{C 0}$ represent the moments of inertia with respect to the axes parallel to the $Y$ axis at the points A and $\mathrm{C}$. After simplifying and dropping the $\delta$ sign, the equations of motion can be obtained

$$
\begin{aligned}
m \ddot{X}= & 4 K_{F X L} X+2 K_{I X S}\left(I_{1}+I_{2}\right) \\
I_{z} \ddot{\theta}= & 4 a^{2} K_{F X L} \theta+2 a K_{I X S}\left(I_{1}-I_{2}\right) \\
m \ddot{Z}= & 4 K_{F Z L} Z+2 K_{F Z S}\left(b_{1} I_{1}+b_{2} I_{2}\right) \phi \\
& +K_{I Z L}\left(I_{A}+I_{B}+I_{C}+I_{D}\right)+2 K_{K Z L} \\
I_{y} \ddot{\phi}= & 2 I_{y}\left(\frac{1}{I_{A 0}}+\frac{1}{I_{C 0}}\right) K_{T Y L} X \\
& +2 a I_{y}\left(\frac{1}{I_{A 0}}+\frac{1}{I_{C 0}}\right) K_{T Y L} \theta \\
& +2 I_{y}\left(\frac{I_{1}}{I_{A 0}}+\frac{I_{2}}{I_{C 0}}\right) K_{T Y S} Z
\end{aligned}
$$




$$
\begin{aligned}
& +2\left[\left(b_{1}^{2}+b_{2}^{2}\right) K_{F Z L}+2\left(b_{1}^{2} I_{1}+b_{2}^{2} I_{2}\right) K_{F Z S}\right] \phi \\
& +2 a I_{y}\left(\frac{I_{1}}{I_{A 0}}-\frac{I_{2}}{I_{C 0}}\right) K_{T Y S} \psi \\
& +2 K_{I Z L}\left[b_{1}\left(I_{A}-I_{B}\right)+b_{2}\left(I_{C}-I_{D}\right)\right]+4 K_{K A S} \\
I_{x} \ddot{\psi}= & 2 a\left(b_{1} I_{1}-b_{2} I_{2}\right) K_{F Z S} \phi+4 a^{2} K_{F Z L} \psi \\
& +a K_{I Z L}\left(I_{A}+I_{B}-I_{C}-I_{D}\right) .
\end{aligned}
$$

The controller will be designed in the next section using these equations.

\section{CONTROLler DesigN}

Based on the modeling process described in the previous section, several assumptions have been made which inevitably will cause some modeling errors. Therefore, the controller to be developed should be robust enough to tolerate these system uncertainties and unmodeling dynamics. Here, the authors prefer to use the adaptive control scheme as a tool to achieve this goal.

\section{A. Plant Model}

In order to obtain a compact controller, the control inputs will be redefined as

$$
\begin{aligned}
& {\left[\begin{array}{l}
u_{1} \\
u_{2}
\end{array}\right]=\left[\begin{array}{cc}
1 & 1 \\
1 & -1
\end{array}\right]\left[\begin{array}{l}
I_{1} \\
I_{2}
\end{array}\right]} \\
& {\left[\begin{array}{l}
u_{3} \\
u_{4} \\
u_{5}
\end{array}\right]=\left[\begin{array}{ccc}
2 & 1 & 1 \\
0 & 1 & -1 \\
2 & -1 & -1
\end{array}\right]\left[\begin{array}{l}
I_{A} \\
I_{C} \\
I_{D}
\end{array}\right]}
\end{aligned}
$$

where $I_{A}=I_{B}$ and the transformation matrices in (3.1) and (3.2) are invertible. Also, to facilitate the design of a singularity-free adaptive controller, the plant model described by $(2.25)$ can be rewritten as

$$
\begin{aligned}
b_{11} \ddot{X}= & a_{11} X+u_{1}+v_{1} \\
b_{22} \ddot{\theta}= & a_{22} \theta+u_{2}+v_{2} \\
b_{33} \ddot{Z}= & a_{33} Z+a_{31} u_{1} \phi+a_{32} u_{2} \phi+u_{3}+v_{3} \\
b_{44} \ddot{\phi}= & a_{41} X+a_{42} \theta+a_{43} u_{1} Z+a_{45} u_{2} Z,+a_{44} \phi+a_{46} u_{1} \phi \\
& +a_{47} u_{2} \phi+a_{48} u_{1} \psi+a_{49} u_{2} \psi+u_{4}+v_{4} \\
b_{55} \ddot{\psi}= & a_{51} u_{1} \phi+a_{52} u_{2} \phi+a_{55} \psi+u_{5}+v_{5}
\end{aligned}
$$

where $b_{i i}>0$ for $i=1 \sim 5, a_{i i}>0$ for $i=1 \sim 2$, and $a_{i i}<0$ for $i=3 \sim 5$. Additionally, $v_{i}, \forall i=1 \sim 5$ do not appear in (2.25), but they are modeled here in order to cancel out the sensor calibration error. These errors occur due to mismatches between the actual neutral points (tracks) and the chosen neutral points (sensors) as shown in Fig. 12, and can be further reduced by calibration of the precision equipment.

In (3.3) and (3.4), $X$ and $\theta$ are decoupled from the other states and can be properly controlled by $u_{1}$ and $u_{2}$. The signals $X, \theta$, $u_{1}$, and $u_{2}$, in (3.5)-(3.7) are measurable, and if the controllers for $X$ and $\theta$ can assure their boundedness, they can be used as known and bounded terms for the controller design of $Z, \phi$ and $\psi$. Rewrite (3.5)-(3.7) as

$$
\mathbf{D}_{B} \ddot{\mathbf{E}}=-\mathbf{D}_{A} \mathbf{E}+\mathbf{U}+\mathbf{D}_{\mathbf{C}} \mathbf{H}+\mathbf{v}
$$

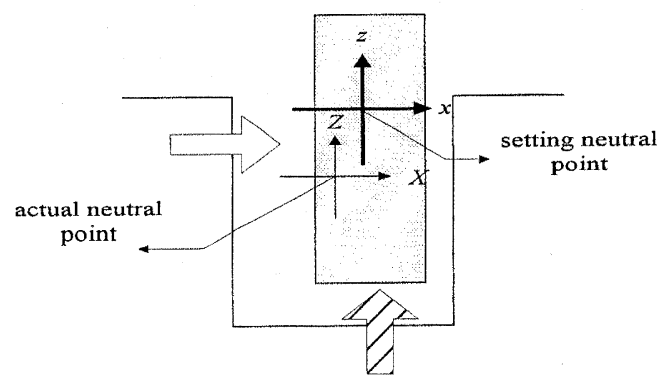

Fig. 12. Mismatch between actual and chosen neutral points.

where

$$
\begin{aligned}
& \mathbf{E}=\left[\begin{array}{lll}
Z & \phi & \psi
\end{array}\right]^{T} \\
& \mathbf{U}=\left[\begin{array}{lll}
u_{3} & u_{4} & u_{5}
\end{array}\right]^{T}, \\
& \mathbf{v}=\left[\begin{array}{lll}
v_{3} & v_{4} & v_{5}
\end{array}\right]^{T} \\
& \mathbf{H}=\left[X, \theta, u_{1} Z, u_{2} Z, u_{1} \phi, u_{2} \phi, u_{1} \psi, u_{2} \psi\right]^{T} \\
& \mathbf{D}_{A}=\operatorname{diag}\left(-a_{33},-a_{44},-a_{55}\right)>0 \\
& \mathbf{D}_{B}=\operatorname{diag}\left(b_{33}, b_{44}, b_{55}\right)>0 \\
& \mathbf{D}_{C}=\left[\begin{array}{cccccccc}
0 & 0 & 0 & 0 & a_{31} & a_{32} & 0 & 0 \\
a_{41} & a_{42} & a_{43} & a_{45} & a_{46} & a_{47} & a_{48} & a_{49} \\
0 & 0 & 0 & 0 & a_{51} & a_{52} & 0 & 0
\end{array}\right] .
\end{aligned}
$$

Based on the above discussion, the controller for lateral modes in (3.3) and (3.4) and vertical modes in (3.8) can be designed, respectively. The forms of (3.3) and (3.4) are the same, so that the symbolic controller derivations are identical. In the following sections, the authors will design the controller only for (3.3). The same results and conclusions can be applied to (3.4).

\section{B. Control Laws}

1) Lateral Control: Due to lack of knowledge of the system parameters, assume that $b_{11}, a_{11}$, and $v_{1}$ are unknown except the sign of $b_{11}$, and then devise the control law as

$$
u_{1}=\hat{b}_{11}\left(2 \lambda_{1} \dot{X}+\lambda_{1}^{2} X\right)+\hat{a}_{11} X+\hat{v}_{1} .
$$

Thus, the closed-loop system becomes

$$
\begin{aligned}
& b_{11} \ddot{X} \\
= & a_{11} X+v_{1}-\hat{b}_{11}\left(\lambda_{1}^{2} X+2 \lambda_{1} \dot{X}\right)-\hat{a}_{11} X-\hat{v}_{1} \\
= & \left(a_{11}-\hat{a}_{11}\right) X-\left(b_{11}-\tilde{b}_{11}\right)\left(\lambda_{1}^{2} X+2 \lambda_{1} \dot{X}\right)+\left(v_{1}-\hat{v}_{1}\right) \\
= & -b_{11}\left(\lambda_{1}^{2} X+2 \lambda_{1} \dot{X}\right)+\tilde{a}_{11} X+\tilde{b}_{11} \zeta_{1}+\tilde{v}_{1}
\end{aligned}
$$

where $\tilde{a}_{11}=a_{11}-\hat{a}_{11}, \tilde{b}_{11}=b_{11}-\hat{b}_{11}, \tilde{v}_{1}=v_{1}-\hat{v}_{1}$ and $\varsigma_{1}=\lambda_{1}^{2} X+2 \lambda_{1} \dot{X}$.

Define

$$
s_{1}=\dot{X}+\lambda_{1} X
$$

Then, (3.10) can be further simplified as

$$
b_{11}\left(\dot{s}_{1}+\lambda_{1} s_{1}\right)=\tilde{a}_{11} x+\tilde{b}_{11} \zeta_{1}+\tilde{v}_{1} .
$$

As in the previous description, control signal $u_{2}$ is designed to control $\theta$ similarly to $u_{1}$. 
2) Vertical Control: Assume $\mathbf{D}_{A}, \mathbf{D}_{B}, \mathbf{D}_{C}$, and $\mathbf{v}$ are unknown, but that the sign of each item in $\mathbf{D}_{B}$ is known. Thus, the control law is chosen as

$$
\mathbf{U}=\hat{\mathbf{D}}_{A} \mathbf{E}-\hat{\mathbf{D}}_{B}\left(\mathbf{G}_{P} \mathbf{E}+\mathbf{G}_{D} \dot{\mathbf{E}}\right)-\hat{\mathbf{D}}_{C} \mathbf{H}-\hat{\mathbf{v}} .
$$

Then, the closed-loop system after (3.13) is substituted into (3.8) becomes

$$
\mathbf{D}_{B}\left[\ddot{\mathbf{E}}+\mathbf{G}_{D} \dot{\mathbf{E}}+\mathbf{G}_{P} \mathbf{E}\right]=-\tilde{\mathbf{D}}_{A} \mathbf{E}+\tilde{\mathbf{D}}_{B} \mathbf{N}+\tilde{\mathbf{D}}_{C} \mathbf{H}+\tilde{\mathbf{v}}
$$

where the estimation errors $\tilde{\mathbf{D}}_{A}=\mathbf{D}_{A}-\hat{\mathbf{D}}_{A}, \tilde{\mathbf{D}}_{B}=\mathbf{D}_{B}-\hat{\mathbf{D}}_{B}$, $\tilde{\mathbf{D}}_{C}=\mathbf{D}_{C}-\hat{\mathbf{D}}_{C}, \tilde{\mathbf{v}}=\mathbf{v}-\hat{\mathbf{v}}$ and $\mathbf{N}=\mathbf{G}_{P} \mathbf{E}+\mathbf{G}_{D} \dot{\mathbf{E}}$.

By choosing $\mathbf{G}_{D}, \mathbf{G}_{P}$ properly, the roots of $\ddot{\mathbf{E}}+\mathbf{G}_{D} \dot{\mathbf{E}}+$ $\mathbf{G}_{P} \mathbf{E}=0$, i.e., $\lambda_{31}, \lambda_{32}, \lambda_{41}, \lambda_{42}, \lambda_{51}$ and $\lambda_{52}$ are all real and negative.

Define

$$
\mathbf{S}=\dot{\mathbf{E}}+\Lambda_{1} \mathbf{E}
$$

where $\Lambda_{1}=\operatorname{diag}\left(\lambda_{31}, \lambda_{41}, \lambda_{51}\right)$, and $\mathbf{S}=\left[\begin{array}{lll}s_{3} & s_{4} & s_{5}\end{array}\right]^{T}$. Then, after some rearrangement (3.14) can be more compactly expressed as

$$
\mathbf{D}_{B}\left(\dot{\mathbf{S}}+\Lambda_{2} \mathbf{S}\right)=-\tilde{\mathbf{D}}_{A} \mathbf{E}+\tilde{\mathbf{D}}_{B} \mathbf{N}+\tilde{\mathbf{D}}_{C} \mathbf{H}+\tilde{\mathbf{v}}
$$

where $\Lambda_{2}=\operatorname{diag}\left(\lambda_{32}, \lambda_{42}, \lambda_{52}\right)$.

\section{Adaptive Laws}

In the previous section, the closed-loop dynamics which involve estimation errors were derived. Now, the adaptive laws will be chosen differently due to effect on the convergence of the errors as follows.

1) Lateral Control: The adaptive laws of lateral control are

$$
\dot{\hat{a}}_{11}=\gamma_{11} X s_{1} \quad \dot{\hat{b}}_{11}=\gamma_{12} s_{1} \varsigma_{1} \quad \dot{\hat{v}}_{1}=\gamma_{13} s_{1}
$$

where $\gamma_{i j}>0$, for $j=1 \sim 3$.

2) Vertical Control: The adaptive laws of vertical control are

$$
\begin{aligned}
\dot{\hat{\mathbf{D}}}_{A} & =-\mathbf{G}_{A} \mathbf{S E}^{T} \\
\dot{\hat{\mathbf{D}}}_{B} & =\mathbf{G}_{B} \mathbf{S N}^{T} \\
\dot{\hat{\mathbf{D}}}_{C} & =\mathbf{G}_{C} \mathbf{S} \mathbf{H}^{T} \\
\dot{\hat{\mathbf{v}}} & =\mathbf{G}_{v} \mathbf{S}
\end{aligned}
$$

where

$$
\begin{aligned}
& \mathbf{G}_{A}=\operatorname{diag}\left(g_{a 1}, g_{a 2}, g_{a 3}\right), \quad \mathbf{G}_{B}=\operatorname{diag}\left(g_{b 1}, g_{b 2}, g_{b 3}\right), \\
& \mathbf{G}_{v}=\operatorname{diag}\left(g_{v 1}, g_{v 2}, g_{v 3}\right), \quad \dot{\hat{a}}_{31}=g_{31}, \quad \dot{\hat{a}}_{32}=g_{32}, \\
& \dot{\hat{a}}_{41}=g_{41}, \quad \dot{\hat{a}}_{42}=g_{42}, \quad \dot{\hat{a}}_{43}=g_{43}, \quad \dot{\hat{a}}_{45}=g_{45}, \\
& \dot{\hat{a}}_{46}=g_{46}, \quad \dot{\hat{a}}_{47}=g_{47}, \quad \dot{\hat{a}}_{48}=g_{48}, \quad \dot{\hat{a}}_{49}=g_{49}, \\
& \dot{\hat{a}}_{51}=g_{51}, \quad \dot{\hat{a}}_{52}=g_{52} \quad g_{i j}>0 \quad \forall i, j .
\end{aligned}
$$

Combining the lateral and vertical control laws, the authors will prove that all the error states are asymptotically stable in the next section.

\section{Stability Analysis}

1) Lateral Control: In order to prove the stability of the closed-loop equation in (3.12), a Lyapunov function candidate $V_{1}$ is chosen as

$$
V_{1}=\frac{1}{2}\left(b_{11} s_{1}^{2}+\frac{1}{\gamma_{11}} \tilde{a}_{11}^{2}+\frac{1}{\gamma_{12}} \tilde{b}_{11}^{2}+\frac{1}{\gamma_{13}} \tilde{v}_{1}^{2}\right) .
$$

Its time derivative can be evaluated as

$$
\dot{V}_{1}=b_{11} s_{1} \dot{s}_{1}+\frac{1}{\gamma_{11}} \tilde{a}_{11} \dot{\tilde{a}}_{11}+\frac{1}{\gamma_{12}} \tilde{b}_{11} \dot{\tilde{b}}_{11}+\frac{1}{\gamma_{13}} \tilde{v}_{1} \dot{\tilde{v}}_{1} \text {. }
$$

Substituting (3.12) and the adaptive laws in (3.17) into (3.20), (3.20) becomes

$$
\begin{aligned}
\dot{V}_{1}= & s_{1}\left(-b_{11} \lambda_{1} s_{1}+\tilde{a}_{11} X+\tilde{b}_{11} \zeta_{1}+\tilde{v}_{1}\right) \\
& +\frac{1}{\gamma_{11}} \tilde{a}_{11} \dot{\tilde{a}}_{11}+\frac{1}{\gamma_{12}} \tilde{b}_{11} \dot{\tilde{b}}_{11}+\frac{1}{\gamma_{13}} \tilde{v}_{1} \dot{\tilde{v}}_{1} \\
= & -b_{11} \lambda_{1} s_{1}^{2}+\tilde{a}_{11}\left(s_{1} X+\frac{1}{\gamma_{11}} \dot{\tilde{a}}_{11}\right)+\tilde{b}_{11}\left(s_{1} \zeta_{1}+\frac{1}{\gamma_{12}} \dot{\tilde{b}}_{11}\right) \\
& +\tilde{v}_{1}\left(s_{1}+\frac{1}{\gamma_{13}} \dot{\tilde{v}}_{1}\right)=-b_{11} \lambda_{1} s_{1}^{2} \leq 0 .
\end{aligned}
$$

From (3.19) and (3.21), it is seen that $V_{1}$ is a suitable Lyapunov function, and, by the Lyapunov stability criteria, $s_{1}, \tilde{b}_{11}$, $\tilde{a}_{11}$, and $\tilde{v}_{1}$ are all bounded, that $s_{1} \in L_{2}$ and in turn, that $\dot{s}_{1} \in L_{\infty}$ by referring back to (3.12). Thus, using Barbalat's Lemma [14], $s_{1}$ is asymptotically stable. From the definition of (3.11), the states $X_{1}$ and $\dot{X}_{1}$ are asymptotically stable, too. Using the same stability analysis, the states $X_{2}$ and $\dot{X}_{2}$ are asymptotically stable.

2) Vertical Control: To prove the stability of the close-loop function (3.16), a Lyapunov function candidate $V_{2}$ is chosen as

$$
\begin{aligned}
V=\frac{1}{2}\left(\mathbf{S}^{T} \mathbf{D}_{B} \mathbf{S}\right. & +\operatorname{tr}\left(\tilde{\mathbf{D}}_{B}^{T} \mathbf{G}_{B}^{-1} \tilde{\mathbf{D}}_{B}\right) \\
& \left.+\operatorname{tr}\left(\tilde{\mathbf{D}}_{A}^{T} \mathbf{G}_{A}^{-1} \tilde{\mathbf{D}}_{A}\right)+\tilde{\mathbf{v}}^{T} \mathbf{G}_{v}^{-1} \tilde{\mathbf{v}}+q\right)
\end{aligned}
$$

where

$$
\begin{aligned}
& q=g_{31}^{-1} \tilde{a}_{31}^{2}+g_{32}^{-1} \tilde{a}_{32}^{2}+g_{41}^{-1} \tilde{a}_{41}^{2}+g_{42}^{-1} \tilde{a}_{42}^{2}+g_{43}^{-1} \tilde{a}_{43}^{2}+g_{45}^{-1} \tilde{a}_{45}^{2} \\
& \quad+g_{46}^{-1} \tilde{a}_{46}^{2}+g_{47}^{-1} \tilde{a}_{47}^{2}+g_{48}^{-1} \tilde{a}_{48}^{2}+g_{49}^{-1} \tilde{a}_{49}^{2}+g_{51}^{-1} \tilde{a}_{51}^{2}+g_{52}^{-1} \tilde{a}_{52}^{2} .
\end{aligned}
$$

Its time derivative is evaluated as

$$
\begin{aligned}
\dot{V}=\left(\mathbf{S}^{T} \mathbf{D}_{B} \dot{\mathbf{S}}+\right. & \operatorname{tr}\left(\dot{\tilde{\mathbf{D}}}_{B}^{T} \mathbf{G}_{B}^{-1} \tilde{\mathbf{D}}_{B}\right) \\
& \left.+\operatorname{tr}\left(\dot{\tilde{\mathbf{D}}}_{A}^{T} \mathbf{G}_{A}^{-1} \tilde{\mathbf{D}}_{A}\right)+\dot{\tilde{\mathbf{v}}}^{T} \mathbf{G}_{v}^{-1} \tilde{\mathbf{v}}+\dot{q}\right)
\end{aligned}
$$

where

$$
\begin{aligned}
\dot{q}= & g_{31}^{-1} \tilde{a}_{31} \dot{\tilde{a}}_{31}+g_{32}^{-1} \tilde{a}_{32} \dot{\tilde{a}}_{32}+g_{41}^{-1} \tilde{a}_{41} \dot{\tilde{a}}_{41}+g_{42}^{-1} \tilde{a}_{42} \dot{\tilde{a}}_{42} \\
& +g_{43}^{-1} \tilde{a}_{43} \dot{\tilde{a}}_{43}+g_{45}^{-1} \tilde{a}_{45} \dot{\tilde{a}}_{45}+g_{46}^{-1} \tilde{a}_{46} \dot{\tilde{a}}_{46}+g_{47}^{-1} \tilde{a}_{47} \dot{\tilde{a}}_{47} \\
& +g_{48}^{-1} \tilde{a}_{48} \dot{\tilde{a}}_{48}+g_{49}^{-1} \tilde{a}_{49} \dot{\tilde{a}}_{49}+g_{51}^{-1} \tilde{a}_{51} \dot{\tilde{a}}_{51}+g_{52}^{-1} \tilde{a}_{52} \dot{\tilde{a}}_{52} .
\end{aligned}
$$

Substituting (3.16) and the adaptive laws in (3.18) into (3.23), (3.23) becomes

$$
\begin{aligned}
& \dot{V}_{2} \\
= & \mathbf{S}^{T}\left(-\mathbf{D}_{B} \Lambda_{2} \mathbf{S}-\tilde{\mathbf{D}}_{A} \mathbf{E}+\mathbf{N}+\tilde{\mathbf{D}}_{C} H+\tilde{\mathbf{v}}\right)+\operatorname{tr}\left(\dot{\tilde{\mathbf{D}}}_{B}^{T} G_{B}^{-1} \tilde{\mathbf{D}}_{B}\right) \\
& +\operatorname{tr}\left(\dot{\tilde{\mathbf{D}}}_{A}^{T} \mathbf{G}_{A}^{-1} \tilde{\mathbf{D}}_{A}\right)+\dot{\tilde{\mathbf{v}}}^{T} \mathbf{G}_{v}^{-1} \tilde{\mathbf{v}}+\dot{q} \\
= & -\mathbf{S}^{T} \mathbf{D}_{B} \Lambda_{2} \mathbf{S}+\operatorname{tr}\left(\mathbf{E} \mathbf{S}^{T} \tilde{\mathbf{D}}_{A}\right)+\operatorname{tr}\left(\mathbf{N} \mathbf{S}^{T} \tilde{\mathbf{D}}_{B}\right) \\
& +\mathbf{S}^{T} \tilde{\mathbf{D}}_{C} H+\mathbf{S}^{T} \tilde{\mathbf{v}}+\operatorname{tr}\left(\dot{\tilde{\mathbf{D}}}_{A}^{T} \mathbf{G}_{A}^{-1} \tilde{\mathbf{D}}_{A}\right)+\operatorname{tr}\left(\dot{\tilde{\mathbf{D}}}_{B}^{T} \mathbf{G}_{B}^{-1} \tilde{\mathbf{D}}_{B}\right) \\
& +\dot{q}+\dot{\tilde{\mathbf{v}}}^{T} \mathbf{G}_{v}^{-1} \tilde{\mathbf{v}}=-\mathbf{S}^{T} \mathbf{D}_{B} \Lambda_{2} \mathbf{S} \leq 0
\end{aligned}
$$



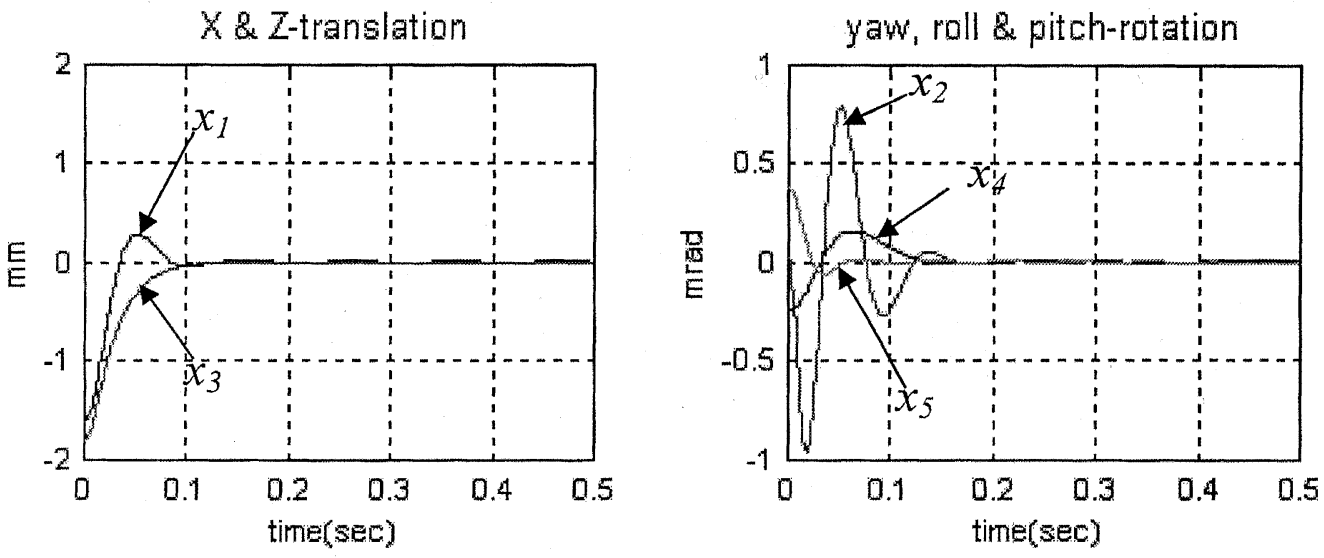

Fig. 13. Simulation result.

TABLE I

SYSTEM DESIGN CONSTANTS

\begin{tabular}{c|c||c|c}
\hline \hline Mass $m$ & $675 \mathrm{~g}$ & Table Area & $200 \mathrm{~mm} \times 270 \mathrm{~mm}$ \\
\hline Stroke & $20 \mathrm{~cm}$ & $a, b_{1}, b_{2}$ & $60,50,70 \mathrm{~mm}$ \\
\hline Turns of levitator & 110 turns & NdFeB Size & $7 \times 25 \times 50 \mathrm{~mm}$ \\
\hline Turns of stabilizer & 160 turns & NdFeB Br & 10000 gauss \\
\hline Diameter of coils & $0.5 \mathrm{~mm}$ & Rubber Magnet Br & 2000 gauss \\
\hline \multicolumn{2}{c|}{$\mathrm{x}_{0}=8 \mathrm{~mm}, \mathrm{y}_{0}=21 \mathrm{~mm}, \mathrm{x}_{\mathrm{p}}=6.5 \mathrm{~mm}, \mathrm{y}_{\mathrm{p}}=25 \mathrm{~mm}, \mathrm{x}_{\mathrm{s}}=7.5 \mathrm{~mm}$} \\
\hline
\end{tabular}

where $\mathbf{D}_{B} \Lambda_{2}$ is the diagonal matrix with positive diagonal terms.

From (3.22) and (3.24), it is seen that $V_{2}$ is a suitable Lyapunov function, and, by the Lyapunov stability criteria, $\mathbf{S}, \tilde{\mathbf{D}}_{A}$, $\tilde{\mathbf{D}}_{B}, \tilde{\mathbf{v}}$, and the parameters which exist in $q$ are all bounded, that $\mathbf{S} \in L_{2}$, and in turn that $\dot{\mathbf{S}} \in L_{\infty}$ by referring back to (3.16). Thus, using Barbalat's Lemma [14], $\mathbf{S}$ is asymptotically stable. From the definition given in (3.15), the states $Z, \dot{Z}, \phi, \dot{\phi}, \psi$, and $\dot{\psi}$ are asymptotically stable.

\section{SIMULATION AND EXPERIMENTAL RESULTS}

\section{A. Simulation Result}

In this section, a simulation is made for the maglev system using the adaptive controller, shown in Fig. 13. The states variables, $x_{i}, i=1 \sim 5$, are, respectively, corresponding to the lateral translation ( $X$ translation), rotation along the $Z$ axis (yawing), vertical translation ( $Z$ translation), rotation along the $Y$ axis (rolling), and rotation along the $X$ axis (pitching). Because the system parameters are difficult to obtain, the system parameters from the design specifications will be calculated. Due to manufacturing and assembling imprecision, these parameters are only rough values. The constants in the system design are listed in Table I.

By simulation results, all the states converge to zero eventually as $t$ goes to infinity, which means the regulating function of the controller is satisfactory. The control parameters for the adaptive controller are set as $\lambda_{1}=100, \lambda_{2}=80, G_{P}=\operatorname{diag}\left[\begin{array}{lll}300 & 200 & 200\end{array}\right]$, and $G_{D}=\operatorname{diag}\left[\begin{array}{lll}30 & 20 & 20\end{array}\right]$. Therefore, the simulation results may well demonstrate the feasibility of the controller design as

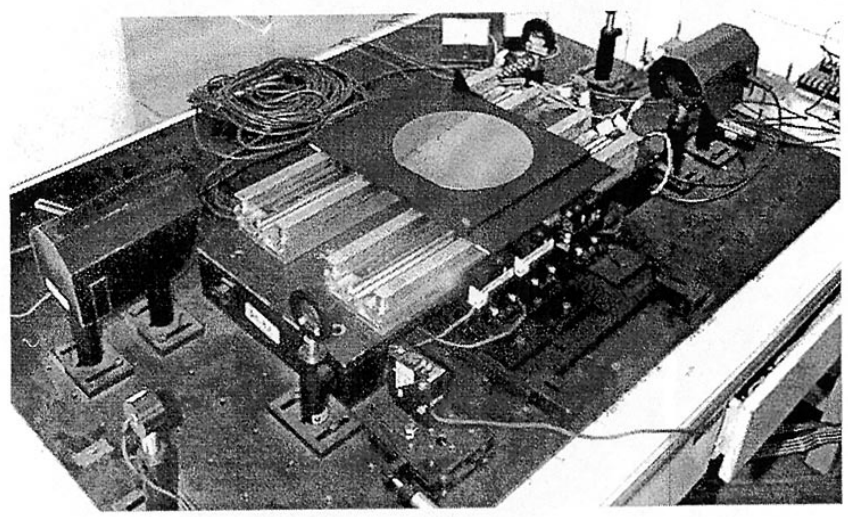

Fig. 14. Photograph of an aspect of the physical system.

well as the consistent physical behavior. In the next section, the tendencies of these simulation results will be used to examine the experimental results later.

\section{B. Experimental Hardware}

The experimental hardware, including the main body, sensor system, driver system, and controller hardware, will be described here. Fig. 14 shows a photograph of the physical setup. A number of experimental results, including the transient and the steady-state responses in different situations, will also be provided in this section to demonstrate the performance of this system with the controller presented in Section III. Based on these results, some important aspects of future research will be presented. 


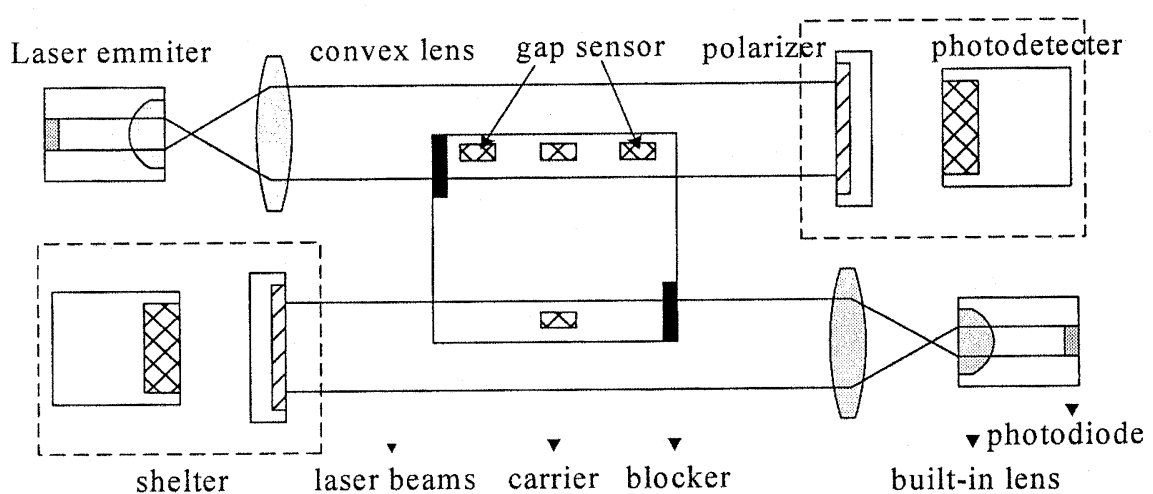

Fig. 15. Final integration in the optical sensing system.
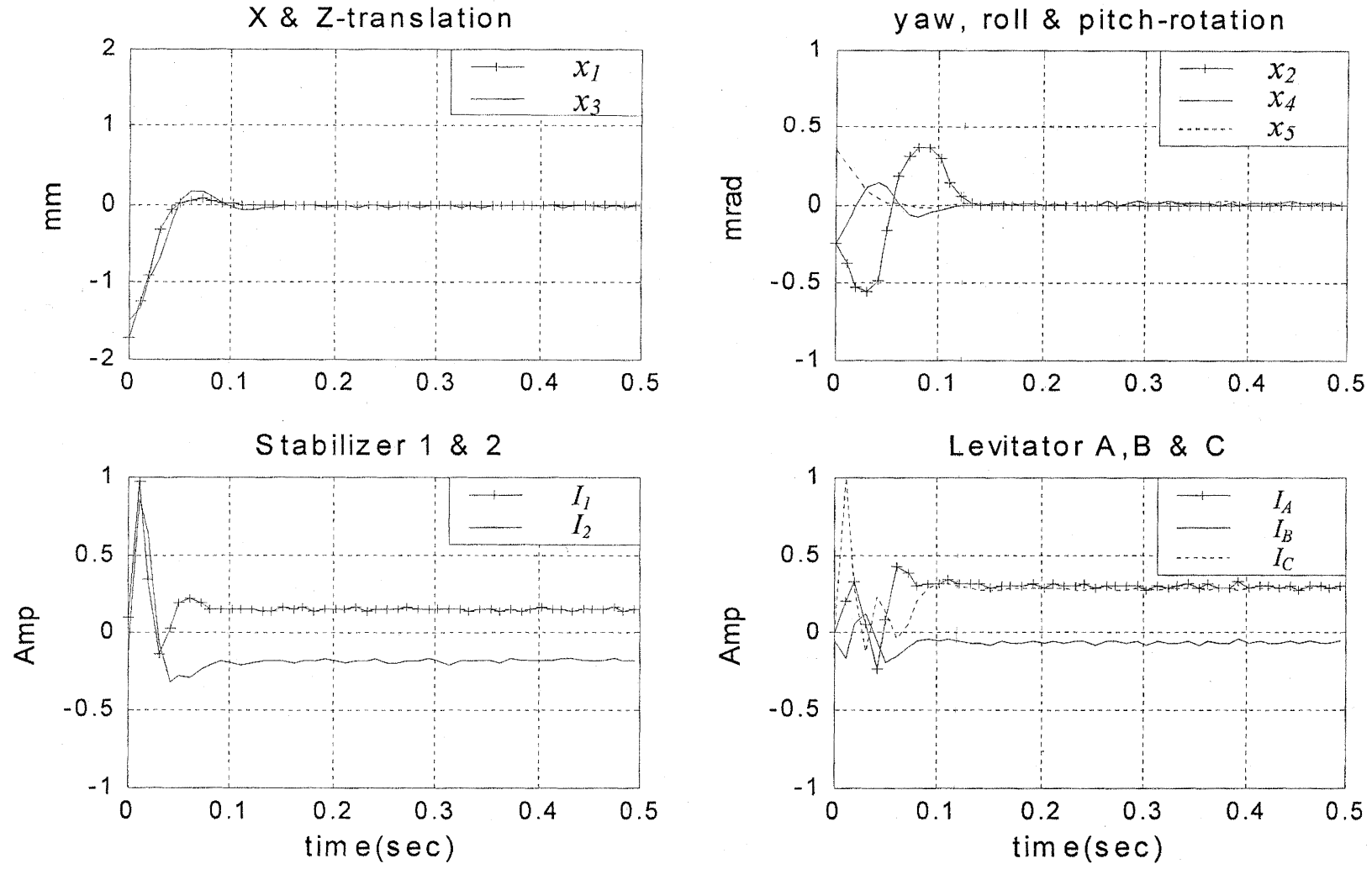

Fig. 16. Transient responses when a full controller is employed (largest translation displacement).

1) Sensor System: This sensor system, which is divided into lateral and vertical sensor subsystems, respectively, provides two translational and three rotational displacement data for the controller. The lateral sensor subsystem is of the optical type as shown in Fig. 15. Two identical emitter-receiver sensor pairs are mounted. Their arrangements are such that the light-emitting directions are parallel but opposite to include special consideration of the system symmetry. The collimation of laser beams is ensured through careful calibration of the convex lens. Two polarizers, which are used to reduce the energy of the laser beams, are placed at positions close to the photodetecters because within the traveling range, the higher the power of the laser beam, the higher the signal-to-noise ratio that can be obtained. In addition, to reduce optical interference, two shelters with openings toward the laser emitters are used to surround the polerizer-photodetector pairs. The photodetecter is chosen to be an ET-4000, manufactured by Electro-Optics Technology, in the U.S. The rise time can be as low as 200 ps whereas the active area is up to $20 \mathrm{~mm}^{2}$, which means this sensor is fast enough and can cover all the traveling range in this application.

The scheme of the vertical sensor subsystem is composed of four gap sensors which are needed to measure for the large travel motion of the carrier. The model number of the vertical gap sensor is Z4W-V25R, and it is manufactured by OMRON Corporation, Japan. The measuring range is $25 \pm 4 \mathrm{~mm}$ and the gap sensor had 1- $\mu \mathrm{m}$ resolution.

2) Driver System and Controller Hardware: The function of drivers in the system is to provide a sufficient amount of currents to the stabilizing and levitating coils. Because the current instead of voltage is used as control input, the current drivers must 

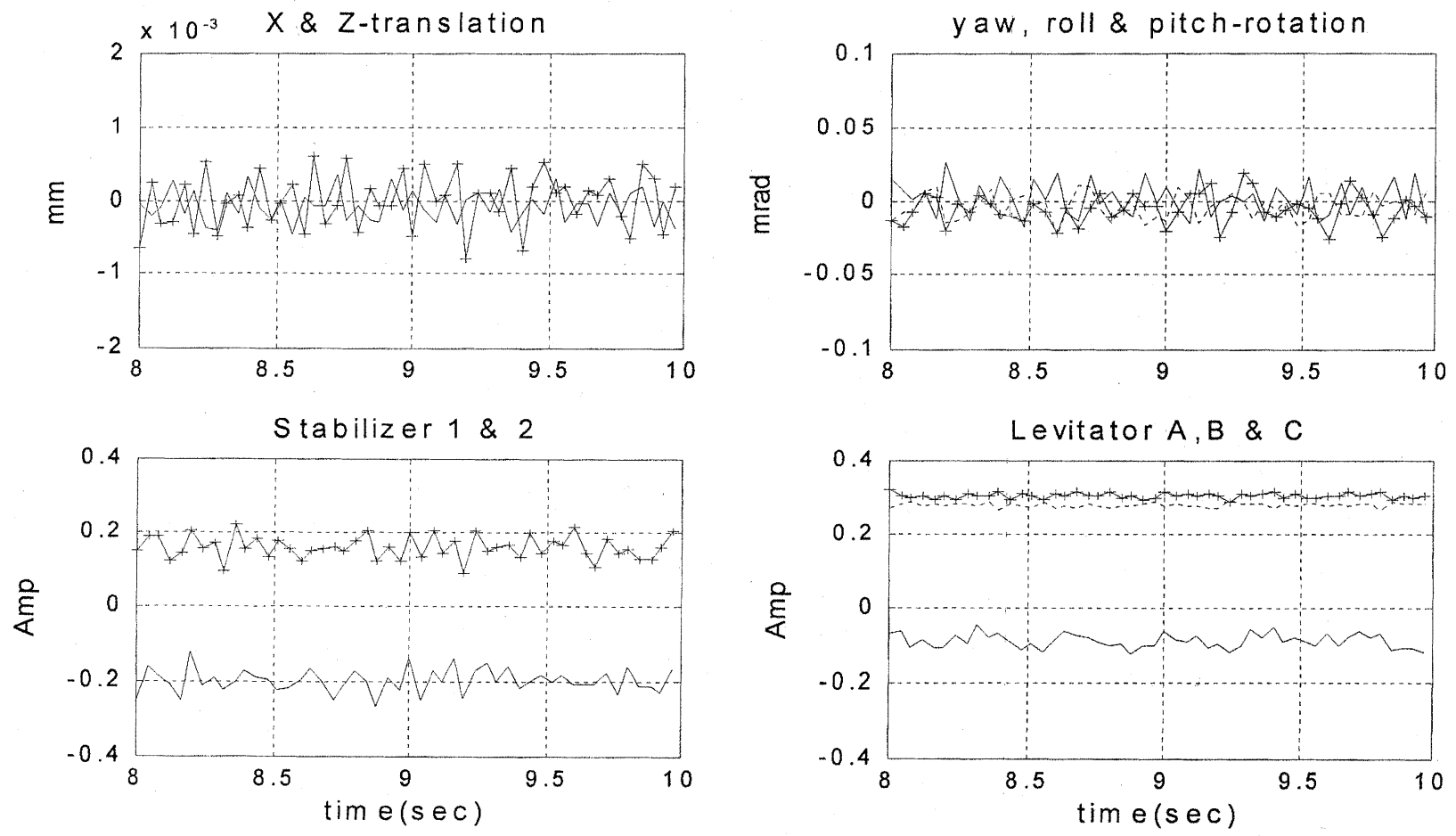

Fig. 17. Steady-state responses when a full controller is employed.
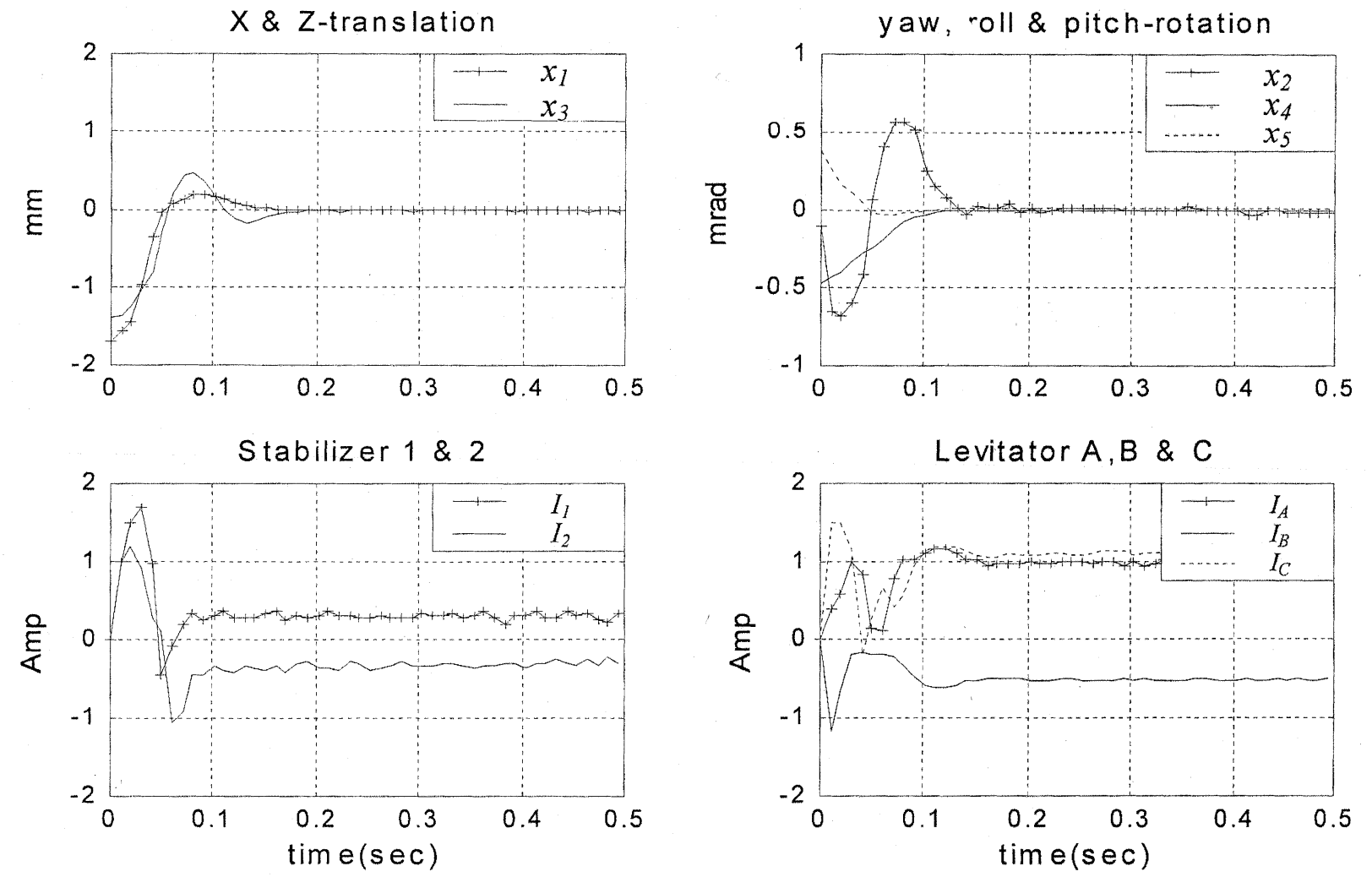

Fig. 18. Responses when a full controller with a load of $250 \mathrm{~g}$ is employed (largest translation displacement).

have enough high bandwidth. The drivers are C0-502-001Q Torque Amplifiers manufactured by CMC Inc., U.S. They are linear drivers designed to be servo drivers for dc motors. The power is $250 \mathrm{~W}, 5 \mathrm{~A}$ for $\pm 50 \mathrm{~V}$ or $10 \mathrm{~A}$ for $\pm 24 \mathrm{~V}$. The current microcomputer is an IBM PC with Pentium microprocessor inside. The clock rate is $150 \mathrm{MHz}$ which allows this research to accomplish a real-time control implementation. Based on the experiential results, a sampling time between $0.1-0.05 \mathrm{~ms}$ leads 

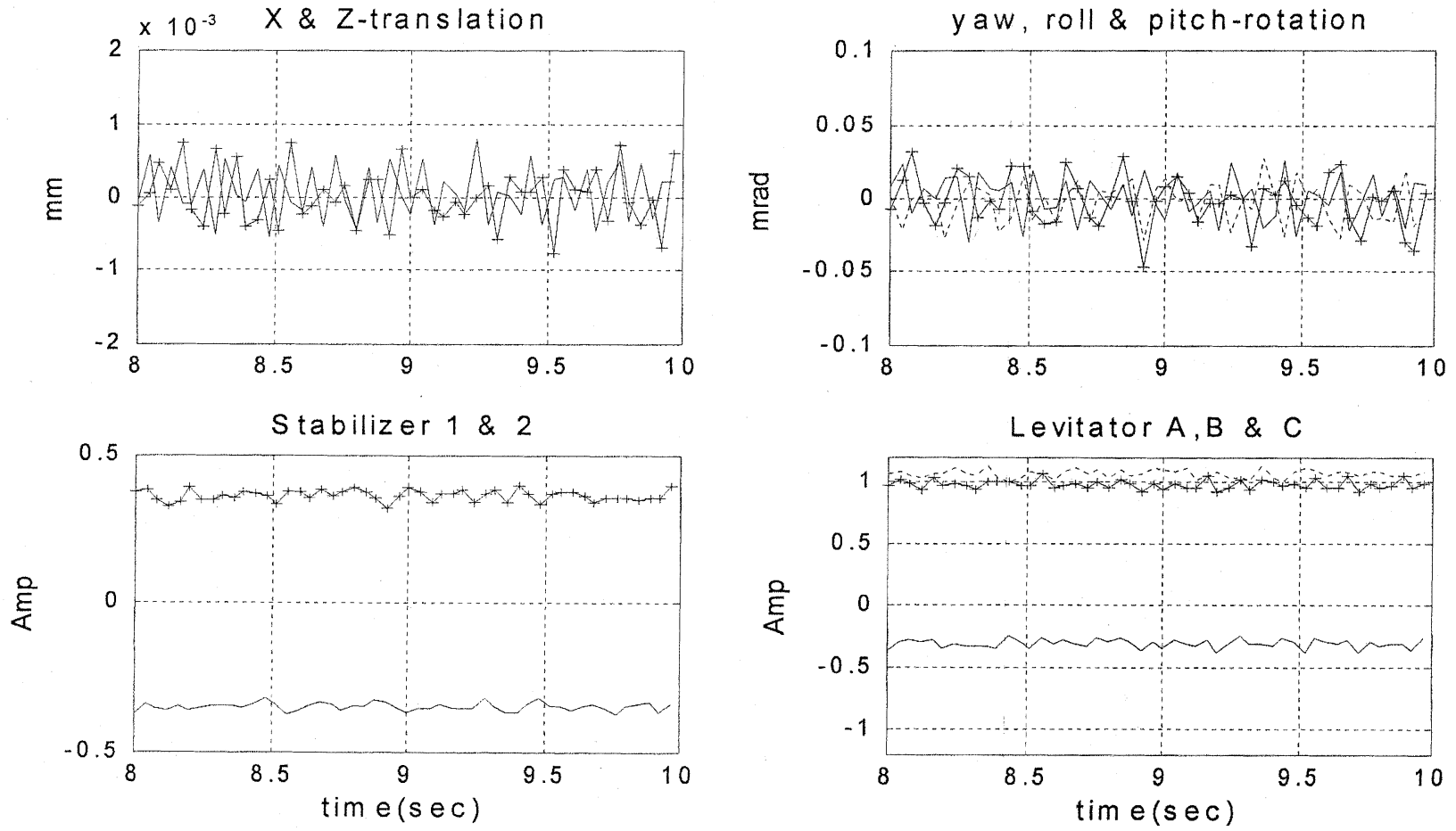

Fig. 19. Steady-state responses when a full controller with a load of $250 \mathrm{~g}$ is employed.

TABLE II

Comparison Between the Hereby Developed System And the System Developed in [9] AND [10]

\begin{tabular}{|c|c|c|c|c|c|c|c|c|c|}
\hline Property & \multicolumn{2}{|c|}{$\begin{array}{c}\text { Wang's } \\
\text { original system }\end{array}$} & \multicolumn{2}{|c|}{$\begin{array}{l}\text { Park's } \\
\text { system }\end{array}$} & \multicolumn{5}{|c|}{ Hereby developed system } \\
\hline $\begin{array}{c}\text { Levitation } \\
\text { magnets }\end{array}$ & \multicolumn{2}{|c|}{ Electromagnets } & \multicolumn{2}{|c|}{ Electromagnets } & \multicolumn{5}{|c|}{ Hybrid magnets } \\
\hline Loading capacity & \multicolumn{2}{|c|}{$150 \mathrm{~g}$} & \multicolumn{2}{|c|}{$150 \mathrm{~g}$} & \multicolumn{5}{|c|}{$250 \mathrm{~g}$ (variable) } \\
\hline $\begin{array}{l}\text { Controller } \\
\text { hardware }\end{array}$ & \multicolumn{2}{|c|}{ Analog circuit } & \multicolumn{2}{|c|}{ Analog circuit } & \multicolumn{5}{|c|}{ Digital microprocessor } \\
\hline $\begin{array}{c}\text { Control } \\
\text { algorithm }\end{array}$ & \multicolumn{2}{|c|}{$\begin{array}{c}\text { Pole placement } \\
\text { control }\end{array}$} & \multicolumn{2}{|c|}{$\begin{array}{c}\text { Lead } \\
\text { compensator }\end{array}$} & \multicolumn{5}{|c|}{ Adaptive control } \\
\hline Performance & $X(\mathrm{~mm})$ & $\theta(\mathrm{mrad})$ & $X(\mathrm{~mm})$ & $\theta$ (mrad) & $X(\mathrm{~mm})$ & $\theta(\mathrm{mrad})$ & $Z(\mathrm{~mm})$ & $\phi(\mathrm{mrad})$ & $\psi(\mathrm{mrad})$ \\
\hline Settling time(sec) & 0.6 & 0.5 & 0.13 & 0.15 & 0.11 & 0.13 & 0.13 & 0.06 & 0.12 \\
\hline Overshoot & 0.1 & 0.43 & 0.2 & 1.7 & 0.1 & 0.33 & 0.15 & 0.19 & 0 \\
\hline Resolution & $2 \times 10^{-3}$ & 0.108 & -- & -- & $1.4 \times 10^{-3}$ & 0.05 & $1.1 \times 10^{-3}$ & 0.05 & 0.03 \\
\hline
\end{tabular}

to better behavior of this system. The type of ADC is a 16-bit high-resolution data acquisition adapter, and the type of DAC is altogether six converting channels with 12-bit resolution each.

\section{Experimental Results and Discussion}

1) Transient Response: Before investigate the transient responses of the closed-loop system, some specifications should be revealed. First, the full $X$-direction translational range is limited to $2 \mathrm{~mm}$, or $\pm 1 \mathrm{~mm}$, whereas the $Z$-axis full rotational range is $0.4 \mathrm{rad}$, or $\pm 0.2 \mathrm{rad}$. On the other hand, the performance of the two cases, respectively, with empty load and 250-g load will be compared.
In Fig. 16, the transient responses for the case with initial maximum translational error are illustrated. From the trajectories of all state, one can see that all of the error signals converge to their steady states within about $0.13 \mathrm{~s}$. In Fig. 18, when the carrier is with $250-\mathrm{g}$ load, the control currents are larger than those in the case with empty load. This is exactly the salient feature of the adaptive controller, which may adjust its controller online to adapt to the change of the environment to ensure the system performance.

2) Steady-State Response: The positioning precision of the system is determined by the steady-state response of the carrier. Figs. 17 and 19 show the system responses around the operating 
points with respect to empty load and the $250-\mathrm{g}$ load. The final precision level up to $1.4 \mu \mathrm{m}$ in translation and $5 * 10^{-2} \mathrm{~m} \mathrm{rad}$ in rotation. It is obvious that the resulting time responses are combinations of a low-frequency component and some high-frequency ones. The low-frequency component, which is around $0.5 \mathrm{~Hz}$, arises from the vibration of the surrounding environment, while the other frequency components are possibly due to the operation of the electromechanical system and interference of the optical sensors.

3) Damping Force: When no control inputs are implied to levitators, the vertical states will be stable because of the existing damping forces. In general, there are three sources of damping forces. They are air damping, an electromagnetic force (EMF), and eddy-current losses resulting from the dynamics magnetic field.

First, it is difficult to make the air damping negligible unless the system is employed in a vacuum environment during operation. Second, the EMF effect is relatively insignificant since all the coils are current controlled in the system. Therefore, the last and most important factor consists of the eddy-current losses, as explained below.

Eddy-current loss is due to heating of the core by circulating currents that are induced in the metallic material core by the varying magnetic field around the turns of the coil. Although the permeability of the material used for the tracks, namely, aluminum, is quite low, the conductivity will inevitably cause eddy-current losses. The kinetic energy will gradually be dissipated due to these energy losses until the system reaches its minimum potential energy.

4) Comparison With Original System: In this section, a comparison between the hereby developed system and original systems [9], [10] is summarized in Table II. By the experiment, the hereby developed system which applied adaptive control obtains performance better than that of the original systems. The advantage of the adaptive controller is that even if the static term is not known exactly, the tuning process will modify the static term to its true value and will result in improved performance.

\section{CONCLUSION}

In this paper, a short-range travel maglev mechanism with high precision has been designed. A repulsive magnetic setup system with four active guiding tracks has been adopted here, which incorporates for advanced technologies in magnetic materials, microelectronics, and optical sensors. The system has been treated as a multi-input/multi-output system, and an adaptive controller has been designed and implemented using a microcomputer. Based on the experimental results, the system's feasibility and effectiveness have been clearly demonstrated. The system performance shows that effects due to nonideal manufacturing and alignment imperfections have been eliminated completely. This implies that improvement in the performance is possible if a more reliable sensing and control are used rather than to use a more expensive hardware setup.

\section{REFERENCES}

[1] R. L. Hollis, S. E. Salcudean, and A. P. Allan, "A six-degree-of-freedom magnetically levitated variable compliance fine-motion wrist: Design, modeling, and control," IEEE Trans. Robot. Automat., vol. 7, pp. 320-332, June 1991.

[2] S. R. Oh, R. L. Hollis, and S. E. Salcudeam, "Precision assembly with a magnetically levitated wrist," in Proc. IEEE Int.Conf. Robotics and Automation, vol. 1, May 1993, pp. 127-134.

[3] B. V. Jayawant, P. K. Sinha, and D. G. Aylwin, "Feedback control system for D.C. electromagnets in passenger-carrying vehicles," Int. J. Control, vol. 24 , no. 5 , pp. 627-639, 1976.

[4] M. Proise, "System concept definition of the Grumman superconducting electromagnetic suspension (EMS) Maglev design," presented at the Maglev'93 Conf., Argonne Nat. Lab., IL, May 19-21, 1993.

[5] E. E. Covert, M. Vlajinac, T. Stephens, and M. Finston, "Magnetic balence and suspension systems for use with wind tunnels," in Progress in Aerospace Science, D. Kuchemann, Ed. New York: Pergamon, 1973, vol. 14.

[6] H. Bleuler, "A survey of magnetic levitation and magnetic bearing types," JSME Int. J., vol. 35, pp. 335-342, 1992.

[7] N. Kosuke and I. Masashi, "A noncontact permanent magnet levitation table with electromagnetic control and its vibration isolation method using direct disturbance cancellation combining optimal regulators," IEEE Trans. Magn., vol. 31, pp. 855-896, Jan. 1994.

[8] D. L. Trumper, S. M. Olson, and P. K. Subrahmanyan, "Modeling and vector control of a planar magnetic levitator," IEEE Trans. Ind. Applicat., vol. 34, pp. 1254-1262, Nov./Dec. 1998.

[9] I. Y. Wang and B. V. Ilene, "A new repulsive magnetic levitation approach using permanent magnets and air-core electromagnets," IEEE Trans. Magn., vol. 30, pp. 1422-1432, July 1994.

[10] K. H. Park, K. Y. Ahn, S. H. Kim, and Y. K. Kwak, "Wafer distribution system for a clean room using a novel magnetic suspension technique," IEEE/ASME Trans. Mechatron., vol. 3, pp. 73-78, Mar. 1998.

[11] M. Y. Chen, K. N. Wu, and L. C. Fu, "Design, implementation and selftuning adaptive control of Maglev guiding system," Mechatron., vol. 10 , pp. 215-237, 2000.

[12] D. T. Greenwood, Principles of Dynamics. Englewood Cliffs, NJ: Prentice-Hall, 1988.

[13] M. H. Nayfeh, Electricity and Magnetism. New York: Wiley, 1985.

[14] K. S. Narendra and A. M. Annaswamy, Stable Adaptive System. Englewood Cliffs, NJ: Prentice-Hall, 1989.

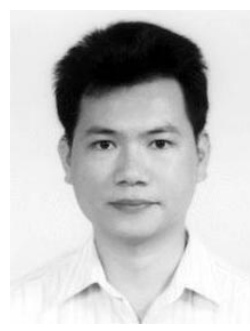

Mei-Yung Chen (S'01) was born in Maju, Taiwan, R.O.C., in 1966. He received the B.S. degree from TamKang University, Taipei, Taiwan, R.O.C., in 1992, and the M.S. degree from Chung Yuan Christian University, Chung-Li, Taiwan, R.O.C., in 1994. He is currently working toward the Ph.D. degree in the Department of Electrical Engineering, National Taiwan University, Taipei, Taiwan, R.O.C.

$\mathrm{He}$ is a Teaching Assistant in the Automatic Control Laboratory, Department of Electrical Engineering, National Taiwan University. His areas of research interest include magnetic levitation technology, positioning and tracking, mechatronics, and control theory and applications.

Mr. Chen is a Member of the Chinese Automatic Control Society, from which he received the Best Student Paper Award in 2001

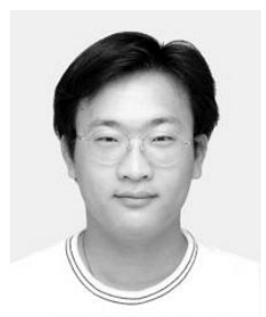

Ming-Jyh Wang was born in Tainan, Taiwan, R.O.C., in 1974. He received the B.S. degree from the Department of Control Engineering, National Chiao-Tung University, HsinChu, Taiwan, R.O.C. in 1996, and the M.S. degree from the Department of Electrical Engineering, National Taiwan University, Taipei, Taiwan, R.O.C., in 1998

Since 1998, he has been with Chung-Shan Institute of Science and Technology, Taoyuan, Taiwan, R.O.C., where he has been involved in the design of airborne computer and multimedia application. His research interests include system integration, adaptive control, and circuit design. 


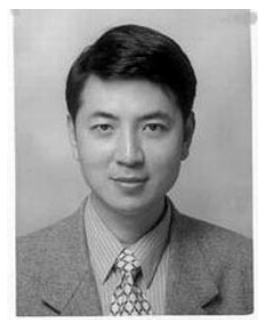

Li-Chen Fu (M'88-SM'02) was born in Taipei, Taiwan, R.O.C., in 1959. He received the B.S. degree from National Taiwan University, Taipei, Taaiwan, R.O.C., in 1981, and the M.S. and Ph.D. degrees from the University of California, Berkeley, in 1985 and 1987, respectively.

Since 1987, he has been a member of the faculty, and is currently a Professor in both the Department of Electrical Engineering and Department of Computer Science and Information Engineering, National Taiwan University, where he also served as the Deputy Director of the Tjing Ling Industrial Research Institute from 1999 to 2001. His research interests include robotics, FMS scheduling, shop floor control, home automation, visual detection and tracking, e-commerce, and control theory and applications.

Prof. Fu is a Senior Member of the IEEE Robotics and Automation and IEEE Automatic Control Societies. He is also a Member of the Boards of the Chinese Automatic Control Society and Chinese Institute of Automation Engineers. During 1996-1998 and 2000, he was appointed as a Member of the IEEE Robotics and Automation Society AdCom and will serve as the Program Chair of the 2003 IEEE International Conference on Robotics and Automation. He received the Excellent Research Award for the period 1990-1993 and Outstanding Research Awards in 1995, 1998, and 2000 from the National Science Council, R.O.C. He also received the Outstanding Youth Medal in 1991, the Outstanding Engineering Professor Award in 1995, and the Best Teaching Award in 1994 from the Ministry of Education, the Ten Outstanding Young Persons Award in 1999 of the R.O.C., the Outstanding Control Engineering Award from the Chinese Automatic Control Society in 2000, and the Lee Kuo-Ding Medal from the Chinese Institute of Information and Computing Machinery in 2000. He has been the Editor of the Journal of Control and Systems Technology and an Associate Editor of the prestigious control journal, Automatica. In 1999, he became the Editor-in-Chief of a new control journal, Asian Journal of Control. 